\title{
Central Banks and Ambiguity ${ }^{1}$
}

\author{
by \\ Willy Spanjers ${ }^{2}$
}

First Version: March 2004

This Version: May 2004

\begin{abstract}
In referring to the 'animal spirits' of investors, Keynes had already pointed to a basic distinction between 'calculable' and 'non-calculable' risk or 'ambiguity'.

The purpose of this paper is to discuss the effects of ambiguity on the public's expectations about inflation and the impact this may have on central bank policy. We consider the case where ambiguity is caused by a lack of predictability in monetary policy. The effects of this loss of predictability are addressed in the setting of a Barro and Gordon type framework, featuring a short run aggregate supply curve. Within this framework we distinguish between 'strategic ambiguity' faced by the public sector and 'state ambiguity' faced by the central bank.

The main results are as follows. Ambiguity about monetary policy can be characterised as a loss of central bank credibility. When the public is pessimistically inclined, its consequences are excessive expectations of inflation and a national income below its natural rate. This result is obtained both in the context of 'rules' and of 'discretion', although the impact of ambiguity is more pronounced in the latter case.

If the public is optimistic with respect to the monetary policy of the central bank, a lack of predictability has no impact on the inflation expectations of the public. The expected rate of inflation is the same as in the absence of ambiguity.

The results are illustrated by considering the effect of initial ambiguity about the European Central Bank, the efforts of the central banks of the EU-accession countries to establish credibility, and the impact of EU-accession on the predictability of their monetary policies. A final illustration is the potential impact of a glorification of the Federal Reserve and its chairman, Alan Greenspan.
\end{abstract}

JEL Classification Codes: D81, E52, E58.

Keywords: Ambiguity, Choquet Expected Utility, Central Bank, Monetary Policy.

\section{INTRODUCTION}

What does it take to establish confidence in a central bank? This question is of major importance for monetary policy makers. But answering it may prove tedious. Firstly, confidence is in the mind of the beholder. It is determined by cognitive processes that seem to be difficult to reconcile with the established paradigms of economic decision making. Even worse, it is not clear what the precise meaning of 'confidence' is in this setting.

\footnotetext{
${ }^{1}$ We would like to thank Paul Auerbach, Subrata Ghatak, the participants in the research seminar of the Department of Economics of Kingston University and the participants in the 3rd Annual Conference of the European Economics and Finance Society in Gdansk for helpful comments and suggestions.

${ }^{2}$ Address for correspondence: Department of Economics, Kingston University, Kingston-upon-Thames, Surrey KT1 2EE, United Kingdom. Email: w.spanjers@kingston.ac.uk
} 
There are models that provide precise interpretations of 'reputation' in the context of incomplete information: they rely on the inability of the public to learn the 'true' preferences and objectives of the central bank. Depending on its 'true' preferences, the central bank may be tempted to deceive the public for a while by pretending to have different preferences. Eventually, of course, the temptation will become too large and the central bank will show its true face. Meanwhile, the longer it resists the temptation, the more probable it will seem to the public that the pretended preferences are its actual preferences and the better its reputation will be. ${ }^{3}$

This hardly seems an appropriate representation of the mechanism that determines presentday confidence in central banks. Indeed, given the decision processes in the leading central banks, it would be hard to imagine that they could succeed in hiding their true preferences from the public for any sustained period of time. We must therefore look for other ways to conceptualise the meaning of 'confidence' in central banks. This paper follows the approach taken in Spanjers (1999b, [16]) to understanding confidence by having a closer look at situations in which it is absent.

We consider the presence of ambiguity to be the main characteristic of situations in which there is a lack of confidence. This leads us to analyse the impact of ambiguity on the choice and effectiveness of monetary policy.

We distinguish between 'calculable' and 'non-calculable' risk and refer to the latter as ambiguity. ${ }^{4}$ Knight considered entrepreneurs to be specialists in dealing with situations of ambiguity, i.e. situations for which there is no relevant experience to guide decision making.

Modern examples of ambiguity include global warming, the BSE-crisis, the Gulf War, the South-East Asian crisis, New Economy technologies and the impact of 9/11. Risk may fail to be calculable for two basic reasons. Firstly, it may not be possible to assign a unique (subjective) probability distribution to different scenarios for the future. Secondly, it may be difficult to associate a unique outcome to each scenario. In either case there is ambiguity.

\footnotetext{
${ }^{3}$ See, e.g. Kreps and Wilson (1982, [9]) and Milgrom and Roberts (1982, [12]).

${ }^{4}$ This distinction goes back to Knight (1921, [8]). He used different terminology but referred to the same concepts.
} 
The next question is how decisions are made in the face of ambiguity. As already indicated by Knight, decisions will depend on the decision maker's attitude with respect to it. Optimists will hope for the best, pessimists will fear the worst. If one insists on referring to subjective probabilities, it represents a situation in which the decision maker's probability assessment depends in a specific way on his choice of action. ${ }^{5}$

The first piece of evidence that decisions under ambiguity may fail to be compatible with the subjective expected utility approach was provided by the famous thought-experiment in Ellsberg (1961, [4]). Schmeidler (1982/1989, [14]) provided an axiomatic foundation for decision making under ambiguity that could match that of subjective expected utility theory. After this breakthrough, economists started to modify their standard analytical tools to deal with ambiguity.

Against this background, let us examine the impact of ambiguity on monetary policy. We consider the interaction of a central bank and its public in a Barro and Gordon (1983, [1]) type of model where the Phillips curve is replaced by a short run aggregate supply curve. ${ }^{6}$ In this framework, the public resents finding itself making false inflation predictions. The central bank dislikes inflation deviating from its optimal level or output being below its natural rate, but it likes output to exceed its natural rate. The analysis here is focused on the impact of strategic ambiguity of the public regarding the monetary policy of the central bank and state ambiguity of the central bank over the position of the short run aggregate supply curve. We consider both a rule based and a discretionary monetary policy. ${ }^{7}$

In the next section, we discuss decision making under ambiguity and the equilibrium concept we use in this paper. In Section 3 we derive the effect of strategic ambiguity about monetary policy on the inflation expectations of the public. We show that ambiguity results

\footnotetext{
${ }^{5}$ See Spanjers (1999a, [15], Chapter 7).

${ }^{6}$ The short run aggregate supply curve can be justified by the presence of sticky wages as in Fischer (1977, [5]), imperfect information, as in Friedman (1968, [6]) and Lucas (1976, [10]), or by sticky prices as in Rotemberg $(1982,[13])$.

${ }^{7}$ Recently, Chprits and Schipper (2003, [2]) have analysed the potential impact of ambiguity for the interaction between a central bank and a trade union in the setting of the original Barro and Gordon model. The aim of our analysis, by contrast, is to provide a framework in which to discuss contemporary central bank policy. Another difference lies in the way ambiguity is treated. Whereas Chprits and Schipper confine their analysis to pessimism, we allow for an optimistic public as well. Finally, we introduce an innovation to the literature on ambiguous beliefs by expressly considering the role of perceived upside and downside risk.
} 
in excessive inflation expectations by a pessimistic public, which negatively affects output. We proceed by including the central bank in the analysis in Section 4. Both for a discretionary and a rule based monetary policy, the effects of ambiguity point in the same direction, but 'rules' dampen its impact. The central bank may face ambiguity about the effectiveness of surprise inflation, which leads to interesting results. For a rule based monetary policy, ambiguity on the effectiveness of surprise inflation only influences monetary policy if the public has less than full confidence in the central bank. More surprisingly, when ambiguity does have an impact, it leads a cautious (i.e. pessimistic) central bank to loosen monetary policy, in order to increase the rate of inflation. In Section 5 we discuss how ambiguity may have influenced the policy of the European Central Bank, how the Eastern enlargement of the EU may increase public confidence in the central banks of the accession countries and, finally, how the glorification of Alan Greenspan may insulate monetary policy from the effects of ambiguity. Concluding remarks are gathered in Section 6.

\section{Decisions under Ambiguity}

In this section we consider decision making under ambiguity using the approach of Choquet Expected Utility. Within this approach, we consider a specific interpretation of the beliefs of the decision makers. After considering individual decision makers, we turn to their interaction, i.e. to equilibrium under ambiguity.

2.1. Decision under ambiguity. Our approach to analysing the impact of ambiguity on decision makers is a generalization of expected utility theory. Under expected utility, the preferences of a decision maker can be represented by a utility function. The utility function assigns each random variable the expected value of the utility each outcome generates. More formally the representation is as follows.

Consider a random variable $x$ with probability distribution $P$, which assigns to each state of nature $s$ an outcome $x(s)$. The utility of each outcome $x(s)$, when it occurs, is evaluated by 
the decision maker according to his von Neumann-Morgenstern utility index $u$ and leads to a utility level of $u(x(s))$. The preferences of the decision maker can be represented by a utility function $U$, which is defined by the expected value of the utility index $u$ over $x$ with respect to $P$, i.e.

$$
U(x):=\mathbb{E}_{P}\{u(x(s))\}
$$

For decision making under ambiguity, this representation is generalized. Apart from a random variable $x$ with an (assessed) probability distribution $P$ and a von Neumann-Morgenstern utility index $u$, other variables determine the utility function $U$. These additional variables include the degree of optimism of the decision maker, $\beta \in[0,1]$, his level of confidence, $\gamma \in[0,1]$, in his assessed probability distribution, the smallest value of the state of nature $\underline{s}$ and the largest value $\bar{s}$ which the decision maker considers to be plausible. ${ }^{8}$ We refer to $\underline{s}$ and $\bar{s}$ as the downside risk and the upside risk of the state of nature, respectively. From these variables the utility function $U$ that represents the preferences of the decision maker is obtained as 9

$$
U(x):=\gamma \cdot \mathbb{E}_{P}\{u(x(s))\}+(1-\gamma) \cdot \beta \cdot\left[\max _{s \in[\underline{s}, s]} u(x(s))\right]+(1-\gamma) \cdot(1-\beta) \cdot\left[\min _{s \in[\underline{s}, \bar{s}]} u(x(s))\right]
$$

This utility function, which represents the preferences of the decision maker, is the sum of three components. The first component reflects the expected utility for the random variable. Its weight equals the level of confidence in the assessed probability distribution. The presence of ambiguity indicates that the outcome of the random variable may deviate from what is indicated by the probability distribution, but it does not indicate whether this deviation is to the advantage or to the disadvantage of the decision maker. The remaining two components reflect the way in which ambiguity enters into his calculations. Their combined weight equals the amount of ambiguity perceived by the decision maker, $(1-\gamma)$.

The degree to which the decision maker is inclined to think that ambiguity leads to an

\footnotetext{
${ }^{8}$ For this particular restriction on the states of nature to have a meaningful interpretation, one must, of course, make certain assumptions about the random variable $x$. A discussion of why this approach is sensible and how it can be formulated in a more general way is beyond the scope of this paper.

${ }^{9}$ We assume that the ambiguity faced by a decision maker is represented by a belief function, which is then combined with his ambiguity attitude to obtain the capacity over which the Choquet integral is taken.
} 
advantageous outcome is represented by his degree of optimism $\beta$. This leads to the second term in the utility function, which contains the von Neumann-Morgenstern utility of the best plausible outcome. The weight of this term is the proportion $\beta$ of the amount of ambiguity $(1-\gamma)$, i.e. $(1-\gamma) \cdot \beta$.

Similarly, $(1-\beta)$ represents the degree to which the decision maker is inclined to think that ambiguity leads to an outcome that is to his disadvantage. This is reflected in the final term of the utility function, which contains the von Neumann-Morgenstern utility value of the worst plausible outcome. This term is weighted by the remaining fraction $(1-\beta)$ of the amount of ambiguity $(1-\gamma)$, hence $(1-\gamma) \cdot(1-\beta)$.

In this paper our main focus is on decision makers who are pessimistically inclined, e.g. for whom $\beta=0$. Furthermore, we restrict attention to probability distributions that concentrate the whole probability mass in a single state of nature $t$. Under these additional assumptions, the utility function of the decision maker reduces to

$$
U(x):=\gamma \cdot u(x(t))+(1-\gamma) \cdot\left[\min _{s \in[s, \bar{s}]} u(x(s))\right]
$$

This utility function assigns the level of confidence $\gamma$ to the utility of the outcome that would 'normally' occur. Because the decision maker is pessimistic, the remaining weight, $(1-\gamma)$, i.e. the level of ambiguity, is assigned to the worst plausible outcome. The associated reasoning of the decision maker could be: 'Although I am rather confident that state $t$ will occur, I am not really sure. As I do not really know what other state may occur instead of $t$, I had better take into account the worst that may plausibly happen'.

2.2. Equilibrium under ambiguity. When considering the interaction of decision makers under ambiguity, one may distinguish between state ambiguity and strategic ambiguity. State ambiguity refers to ambiguity about the environment in which the interaction takes place. It should be addressed in a similar way to interaction in a stochastic environment, the only difference being that the impact of ambiguity is taken into account. ${ }^{10}$ For state ambiguity,

\footnotetext{
${ }^{10}$ I.e. the expected utility is obtained by applying the Choquet integral to the appropriate representation of the beliefs.
} 
there is no reason to refrain form using the usual game-theoretic equilibrium concepts, since the only impact of ambiguity is to change the objective functions of the players.

In a game, decision makers may also face a different type of ambiguity. It does not refer to the environment in which decisions are made, but rather to the choice of strategies by the other players. We refer to such ambiguity as strategic ambiguity. When considering strategic ambiguity, there may be good reasons to apply equilibrium concepts that are specifically developed to deal with ambiguity about the strategy choices of other players. Such concepts have been introduced in Dow and Werlang (1994, [3]), Marinacci (2000, [11]) and Kelsey and Spanjers $(2004,[7])$.

These equilibrium concepts are appropriate for some situations of strategic ambiguity, but not for all. They look for combinations of non-additive beliefs and strategies that are mutually consistent. Usually, this leads to a multitude of equilibrium beliefs and equilibrium strategies. As each of the many equilibria is internally consistent, the model offers no obvious way to select any specific equilibrium. For our present purposes, these equilibrium concepts are of little use because of their inherent indeterminacy. We do want to consider strategic ambiguity, but on the other hand, we wish to find a unique equilibrium. This goal can be achieved by allowing for ambiguity with respect to the strategy choices of the players, but by assuming that the corresponding beliefs have a very particular shape. The reason for so doing is that in most applications ambiguity takes a specific form: it is not just arbitrarily picked from a large choice of possibilities. More to the point, we believe that ambiguous beliefs are the outcome of a cognitive process, for now unspecified. This process not only determines the degree of familiarity with a given situation, and thus the 'amount' of ambiguity that is experienced, but also puts bounds on what are considered plausible values of the ambiguous variables.

One may want to identify these boundaries with the more familiar concepts of upside risk and downside risk, referring here to the maximum and minimum values that may plausibly be obtained. In this context, learning about an ambiguous variable consists of both finding more reliable values for the upside and the downside risk and of reducing the amount of 
ambiguity faced. One practical way of promoting this process of learning is by first observing how the ambiguous variable behaves when it is not interfered with. Once this experience is gathered, deliberate experiments may be used to learn more about its behaviour in extreme circumstances.

In the absence of more solid theories as to how (strategic) ambiguity arises and develops, we consider the beliefs that describe it as an exogenously given characteristic of each player. In doing so, the mathematical treatment of strategic ambiguity becomes identical with the treatment of state ambiguity parameterized by the choices of strategies by the other players. The conceptual difference, of course, remains.

The consequence of all this is that, even though we consider games in which players face both state and strategic ambiguity, the methods for determining Nash equilibrium and backward induction can still be applied. The only difference is that players have somewhat unusual objective functions.

In the remainder of the paper, a Nash equilibrium in pure strategies is considered in the case of discretionary monetary policy. For this situation, the best response functions of the public and the central bank are derived and equilibrium is obtained for the strategies that are mutual best responses. To obtain the equilibrium for a rule based monetary policy, backward induction is applied. Here the central bank announces its optimal strategy, assuming that the public reacts according to its best response function.

In the next section we derive the best response function of the public before we derive equilibrium monetary policy in Section 4.

\section{The Public}

In this simple model, we focus on the public's expectations of inflation. This makes sense, since these expectations determine a wide range of economic decisions by consumers, firms and the government. These economic decisions influence the output of the economy, in particular the deviation of the level of output from its natural rate. 
For the purpose of simplification, we assume that the actual inflation rate is determined by the monetary policy of the central bank. This is not to say there are no other or, indeed, more important determinants of the rate of inflation. Rather, we choose not to consider them. We also abstract from any interactions amongst the public that may influence the level of national income. For example, we disregard the possibility of multiple equilibrium levels of expenditure and output and the coordination problems that result from this.

Under these assumptions, each decision maker in the public faces the same decision problem, that is to say making the best possible prediction $\pi^{e}$ of the actual inflation rate $\pi$ chosen by the central bank. We assume he does so by maximizing a quadratic objective function:

$$
\max _{\pi^{e}}-\left(\pi-\pi^{e}\right)^{2}
$$

As is well-known, for any actual rate of inflation $\pi$ this results in

$$
\pi^{e}(\pi)=\pi
$$

as the best response function of the public.

Strategic ambiguity faced by the public. The public may, however, face strategic ambiguity regarding the decision of the central bank. Such beliefs may be interpreted as stating that the public expects the central bank to choose a particular inflation rate, but it is not quite sure if the central bank may not, in some unpredictable way, deviate from this action. Obviously, the more confidence the public has in the central bank, the less emphasis it will put on potential unpredictable deviations. We refer to this as strategic ambiguity, as it relates to the central bank's choice of strategy rather than to the exogenous economic environment, i.e. to the state of nature.

The confidence of the public that the central bank acts as anticipated is denoted by the level of confidence $\gamma \in[0,1]$, where $(1-\gamma)$ denotes the amount of strategic ambiguity. Assuming that the public is pessimistically inclined, its decision problem becomes

$$
\max _{\pi^{e} \in[\underline{\pi}, \bar{\pi}]} \gamma\left[-\left(\pi-\pi^{e}\right)^{2}\right]+(1-\gamma)\left[\min _{\pi \in[\pi, \pi]}-\left(\pi-\pi^{e}\right)^{2}\right]
$$


Here the maximal plausible downside risk and the maximal plausible upside risk with respect to the actual rate of inflation enter into the considerations of the public as psychological points of reference. The downside risk of inflation is represented by the minimal plausible inflation rate $\underline{\pi}$, the upside risk by $\bar{\pi}$. Obviously, these estimations of upside and downside risk depend both on the particular situation under consideration and on the history from which it arises.

We assume that the upside risk of the inflation rate $\pi$ exceeds the downside risk for the expected rate of inflation $\pi^{e}$, which seems a plausible assumption to make. From this, we obtain as the optimal or best response inflation expectation of the public ${ }^{11}{ }^{12}$ :

$$
\pi^{e}(\pi, \gamma)=\gamma \pi+(1-\gamma) \bar{\pi}
$$

Proposition 1. For a pessimistically inclined public, a decrease in the level of confidence in the central bank leads to a higher expected rate of inflation.

Short Run Aggregate Supply. We may use a simple short run aggregate supply curve to relate the actual and expected rates of inflation to the level of output:

$$
y\left(\pi, \pi^{e}\right)=y^{n}+a\left(\pi-\pi^{e}\right),
$$

where $a$ denotes the effectiveness of surprise inflation. It should be noted, however, that the effectiveness of surprise inflation also represents the impact on output of incorrect inflation expectations by the public!

\footnotetext{
${ }^{11}$ When the downside risk with respect to expected inflation exceeds the upside risk, i.e. $\pi^{e}>\frac{1}{2}(\underline{\pi}+\bar{\pi})$, we obtain$$
\pi^{e}(\pi, \gamma)=\gamma \pi+(1-\gamma) \underline{\pi}
$$

This case occurs whenever

$$
\pi>\frac{1}{2 \gamma} \bar{\pi}+\left(1-\frac{1}{2 \gamma}\right) \underline{\pi} .
$$

${ }^{12}$ For an optimistic public, for each $\pi^{e}$ the best plausible outcome is obtained by solving

$$
\max _{\pi \in[\underline{\pi}, \bar{\pi}]}-\left(\pi-\pi^{e}\right)^{2}
$$

which leads to the decision problem

$$
\max _{\pi^{e} \in[\pi, \pi]} \gamma\left[-\left(\pi-\pi^{e}\right)^{2}\right] .
$$

Using the latter, the best response expected inflation for an optimistic public follows as

$$
\pi^{e}(\pi, \gamma)=\pi
$$


For any given choice of actual inflation rate $\pi$ by the central bank, the inflation expectation $\pi^{e}$ leads to a level of output

$$
y=y^{n}+a(1-\gamma)(\pi-\bar{\pi})<y^{n} .
$$

Proposition 2. For a pessimistically inclined public, strategic ambiguity over the given monetary policy $\pi$ depresses output below its natural rate.

\section{The Central Bank}

The next question we address is how a lack of confidence in the central bank affects the behaviour of the central bank. Along the lines set out by Barro and Gordon (1983, [1]), we consider two cases: the case in which the monetary policy of the central bank follows some kind of rules and the case in which the central bank chooses the inflation rate as it pleases, i.e. at its discretion. We first consider discretionary monetary policy. Thereafter the situation in which the central bank is committed to its announced monetary policy, the rule based case, is analysed.

4.1. Discretion. When the central bank is not committed to any type of target, it may choose its monetary policy to fit its own objectives. At the same time that the central bank determines its monetary policy, the public determines its inflation expectations. In the absence of ambiguity, the resulting interaction can be described by a Nash equilibrium in pure strategies in the appropriate normal form game. ${ }^{13}$

No ambiguity by the central bank. We assume that there is a certain level of inflation $\pi^{n}$ that the central bank considers most appropriate for the economy as a whole. Furthermore,

\footnotetext{
${ }^{13}$ Alternatively, one may assume that the central bank decides on the actual level of inflation after the public formed its inflation expectations. The public, of course, takes this into account in determining its inflation expectations. In the absence of ambiguity and for the objective function of the public considered in this paper, both approaches lead to the same outcome.

The reason for this coincidence of outcomes is that the best possible indifference curve of the public coincides with its best response function. In the presence of ambiguity, as for different specifications of the public's objective function, these competing approaches lead to different outcomes.
} 
the central bank would prefer a level of output above its natural rate $y^{n}$ and dislikes it being less. Thus, the objective of the central bank is

$$
\max _{\pi} b\left(y\left(\pi, \pi^{e}\right)-y^{n}\right)-\left(\pi-\pi^{n}\right)^{2}
$$

Taking the short run aggregate supply curve into account, this expression becomes

$$
\max _{\pi} b a\left(\pi-\pi^{e}\right)-\left(\pi-\pi^{n}\right)^{2}
$$

For given inflation expectations $\pi^{e}$ of the public, the optimal choice of inflation rate by the central bank, i.e. its best response, is

$$
\pi\left(\pi^{e}\right)=\pi^{n}+\frac{1}{2} b a
$$

The resulting Nash equilibrium between the private sector and the central bank is characterized by the intersection of the best response curves, which yields:

- $\pi=\pi^{n}+\frac{1}{2} b a$,

- $\pi^{e}=\gamma\left(\pi^{n}+\frac{1}{2} b a\right)+(1-\gamma) \bar{\pi}>\pi$

- $y=y^{n}+a(1-\gamma)\left[\pi^{n}-\bar{\pi}\right]<y^{n}$.

This result is illustrated in Figure 1. 14

State ambiguity of the central bank. Next we address the question of how ambiguity faced by the central bank affects these results. We focus on ambiguity concerning the position of the short run aggregate supply curve. We refer to this type of ambiguity as state ambiguity, as it concerns the exogenous economic environment, i.e. the state of nature, rather than the choice of strategy by the public. We consider ambiguity over the natural rate of output and ambiguity with respect to the effectiveness of surprise inflation.

\footnotetext{
${ }^{14}$ As a point of reference, the corresponding result for a central bank with the objective

$$
\max _{\pi}-b\left(y\left(\pi, \pi^{e}\right)-y^{n}\right)^{2}-\left(\pi-\pi^{n}\right)^{2}
$$
}

is depicted in Figure 2. 
Ambiguity with respect to the natural rate of output may be a very real problem for central banks. However, as can be seen from the above, the natural rate of output does not occur in the decision problem of the central bank. Therefore, ambiguity over the natural rate of output has no impact on the central bank's policy. It should be noted that a more complicated specification of the short run aggregate demand curve may well allow the natural rate to enter the decision problem of the central bank. In such a case, ambiguity with respect to the natural rate may influence monetary policy.

As the effectiveness of surprise inflation does enter into the decision problem of the central bank, one may expect ambiguity with respect to it to have an impact on monetary policy. Let $\delta$ denote the level of confidence of the central bank in its estimate $a$ of the effect of surprise inflation. The belief of the central bank should be interpreted as stating: 'We think $a$ correctly represents the effectiveness of surprise inflation, but we are not sure. However, we have no clear indication what alternative value of $a$ might apply.' If the central bank is pessimistically inclined with respect to this variable, its decision problem becomes:

$$
\max _{\pi} \delta\left[b a\left(\pi-\pi^{e}\right)-\left(\pi-\pi^{n}\right)^{2}\right]+(1-\delta)\left[\min _{\widetilde{a} \in[\underline{a}, \bar{a}]} b \widetilde{a}\left(\pi-\pi^{e}\right)-\left(\pi-\pi^{n}\right)^{2}\right] .
$$

The upside risk and the downside risk regarding the effectiveness of surprise inflation now come into play. In the above expression $\underline{a}$ denotes the lowest value of $a$ which the central bank still considers plausible, $\bar{a}$ denotes the highest such value. Provided that $\pi^{e}>\pi$, as derived in the previous section, the minimum is obtained for $\bar{a}$ and the decision problem reduces to ${ }^{15}$

$$
\max _{\pi}[\delta a+(1-\delta) \bar{a}] b\left(\pi-\pi^{e}\right)-\left(\pi-\pi^{n}\right)^{2}
$$

The optimal choice of inflation rate by the central bank for given expectations $\pi^{e}$ of the public and level of confidence $\delta$ is

$$
\pi\left(\pi^{e}, \delta\right):=\pi^{n}+\frac{1}{2}[\delta a+(1-\delta) \bar{a}]>\pi\left(\pi^{e}, 1\right)
$$

Proposition 3. In presence of ambiguity over the effectiveness of surprise inflation, a cautious central bank chooses a higher level of inflation than in its absence.

\footnotetext{
${ }^{15}$ For $\pi^{e}<\pi$ the minimum is obtained for $\underline{a}$ and the expression changes accordingly.
} 
The intuition of this result is as follows. If the expectation of inflation by the public is higher than the inflation rate chosen by the central bank, this depresses output below its natural rate. This is bad for the central bank, as it also cares about the level of output. In the presence of ambiguity, additional weight is placed on the worst state. For the central bank, the worst state is that the excessive inflation expectations have a large impact on the level of output. But this occurs when the impact of deviations of actual inflation from expected inflation have a large impact on the level of output, i.e. when surprise inflation is most effective.

To counteract this potentially harmful effect of excessive inflation expectations, the central bank is prepared to accept a larger deviation of the inflation rate from its preferred value $\pi^{n}$. This causes the central bank to increase the rate of inflation.

The result is illustrated in Figure 3. ${ }^{16}$

4.2. Rules. The major contemporary central banks in essence follow inflation targets. Therefore, one may argue, the most relevant case is not central bank discretion. One should rather focus on the question of how ambiguity affects the behaviour of a central bank that follows a rule based monetary policy.

In order to consider the comparative static effects of ambiguity on central bank behaviour, we introduce some flexibility into the rule based framework. We maintain what we consider to be the basic property of rule based monetary policy: the central bank implements whatever inflation rate it announces. We deviate from the usual approach, however, by allowing the central bank to announce any inflation rate it seems fit. The announced inflation rate may depend both on the strategic ambiguity of the public concerning the central bank's behaviour and on the state ambiguity faced by the central bank itself.

No ambiguity by the central bank. As before, the objective of the central bank is

$$
\max _{\pi} b\left(y\left(\pi, \pi^{e}\right)-y^{n}\right)-\left(\pi-\pi^{n}\right)^{2}
$$

Since the announcement of the central bank is, in principle, credible, the central bank can predict how its announcement influences the inflation expectations of the public. Anticipating

\footnotetext{
${ }^{16}$ The corresponding result for an optimistic public and a pessimist central bank is illustrated in Figure 6 .
} 
that the inflation expectations of the public are

$$
\pi^{e}(\pi, \gamma)=\gamma \pi+(1-\gamma) \bar{\pi}
$$

as derived in Section 3, the decision problem of the central bank becomes

$$
\max _{\pi} b a(1-\gamma)(\bar{\pi}-\pi)-\left(\pi-\pi^{n}\right)^{2} .
$$

From this it follows that the central bank's optimal choice of the actual inflation rate $\pi$ for a given level of confidence $\gamma$ on the part of the public is

$$
\pi(\gamma)=\pi^{n}+\frac{1}{2} b a(1-\gamma)>\pi^{n}
$$

The equilibrium for a rule based monetary policy is described by:

- $\pi=\pi^{n}+\frac{1}{2} b a(1-\gamma)$

- $\pi^{e}=\gamma \pi+(1-\gamma) \bar{\pi}=\gamma \pi^{n}+(1-\gamma) \bar{\pi}+\frac{1}{2} b a(1-\gamma) \gamma$

- $y=y^{n}+a(1-\gamma)\left[\pi^{n}-\bar{\pi}+\frac{1}{2} b a(1-\gamma)\right]$.

Therefore, even when the central bank is bound by the level of inflation it announces, a lack of confidence by the private sector leads to excessive inflation. There are, however, two noteworthy differences between the rule based case and that of a discretionary monetary policy.

Firstly, in the case of 'rules', the deviation of the actual inflation rate $\pi$ from the optimal rate $\pi^{n}$ is less than under 'discretion'. The impact of ambiguity on output is also less.

Secondly, the level of confidence influences the central bank's choice of inflation rate. In the case of discretion, the inflation rate is independent of the strategic ambiguity faced by the public.

This result is illustrated in Figure 4.

State ambiguity of the central bank. How does state ambiguity with respect to the location of the short run aggregate supply curve affect monetary policy when the central bank is bound by rules? 
As before, ambiguity about the natural rate of output has no impact on monetary policy. So what is the impact of ambiguity with respect to the effectiveness of surprise inflation? The level of confidence of the central bank in the value of $a$ is denoted by $\delta$. The decision problem of a central bank that faces a public with a level of confidence $\gamma$ is now given by

$$
\begin{aligned}
& \max _{\pi} \delta\left[-b a(1-\gamma)(\bar{\pi}-\pi)-\left(\pi^{n}-\pi\right)^{2}\right] \\
& +(1-\delta)\left[\min _{\tilde{a} \in[\underline{a}, \bar{a}]}-b \widetilde{a}(1-\gamma)(\bar{\pi}-\pi)-\left(\pi^{n}-\pi\right)^{2}\right]
\end{aligned}
$$

Assuming that $\bar{\pi}-\pi \geq 0$, the minimum in the second part of the expression is obtained for $\bar{a}$, as in the case of discretion. Therefore, the decision problem reduces to

$$
\max _{\pi}-b[\delta a+(1-\delta) \bar{a}](1-\gamma)(\bar{\pi}-\pi)-\left(\pi^{n}-\pi\right)^{2} .
$$

The optimal choice of inflation rate by the central bank, for given levels of confidence $\gamma$ and $\delta$, is

$$
\pi(\gamma, \delta):=\pi^{n}+\frac{1}{2}[\delta a+(1-\delta) \bar{a}] b(1-\gamma) \geq \pi^{n}
$$

Proposition 4. Suppose that the central bank and the public are both pessimistically inclined. Provided that the private sector faces ambiguity $(\gamma<1)$, the ambiguity faced by the central bank $(\delta<1)$ increases the inflation rate it chooses compared to the case in which it faces no ambiguity $(\delta=1)$.

If the public has full confidence in the central bank $(\gamma=1)$, then the level $(1-\delta)$ of state ambiguity of the central bank does not affect the level of output.

The result is illustrated in Figure 5. ${ }^{17}$

The ambiguity faced by the central bank only has an impact on the level of output when the private sector faces ambiguity about the central bank. If the private sector has full confidence in the announcements of the central bank, its expected rate of inflation equals the announced

\footnotetext{
${ }^{17}$ The corresponding result for an optimistic public and a pessimistic central bank is depicted in Figure 7.
} 
level and output remains at its natural rate. The state ambiguity faced by the central bank affects the output of the economy only to the extent that the expected rate of inflation differs from the inflation rate announced by the central bank.

From this we may conclude that a rule based monetary policy is better suited to cope with ambiguity when the private sector and the central bank are inclined to pessimism.

\section{Examples of Central Banks and Ambiguity}

5.1. The European Central Bank. Considering the introduction of the Euro, the public faced some strategic ambiguity with respect to the monetary policy of the European Central Bank, as its monetary policy was untested. This may have led to a level of output below its natural rate, even if the European Central Bank cared about the level of output in the Eurozone. Increasing the transparency of decision making on monetary policy was not an option. Public pressure on the national central banks to vote in accordance with the state of their national economies would have increased, leading to more, rather than less, ambiguity.

One may well argue that the basic impact of the Stability and Growth Pact was to enhance confidence in the monetary policy of the European Central Bank. Because of the pact, there was less reason to believe that monetary policy would bend to the interests of fiscally irresponsible governments. It seems to have been a prudent decision of the ECB to maintain a steady hand with respect to interest rates. Even when economic circumstances may at times have allowed for a more activist monetary policy, the confidence enhancing effect of its steady policies may, in its early years, have been more important. It may also have decreased the upside and downside risk perceived by the public. Now that the ECB has enhanced its credibility, the Stability and Growth Pact seems to have fulfilled its purpose and may have become obsolete with regard to the present group of Eurozone countries.

5.2. Central Banks in Central and Eastern Europe. After disposing of their communist governments and starting on the way to becoming market economies, the public in the central and eastern European countries faced much ambiguity about their central banks and 
their monetary policies due to the lack of relevant past experience with these central banks. Tough monetary policy in the early days may have helped to establish confidence in the central banks. The (prospect of) membership of the EU may also have had a confidence enhancing effect, as it reduced the scope for governments and central banks to pursue unpredictable monetary policies. According to the analysis of this paper, this gain in confidence may lead to lower inflation rates and output levels closer to their natural rate.

5.3. Glorification of Alan Greenspan. A final example intends to highlight the impact of optimism by the public regarding the central bank. Consider the Federal Reserve and the reputation it has established in recent years, with the glorification of Alan Greenspan. On this basis, one may argue that, in the present climate, the mood of the US public regarding its central bank comes close to one of optimism. When the public is optimistic regarding the actions of the central bank, ambiguity has no impact on the expected rate of inflation. An optimistic public associates ambiguity with the best, rather than with the worst plausible outcome. For the public, the best outcome occurs when the actual rate of inflation equals the rate of inflation it expects. An optimistically inclined public thus insulates the economy from a loss in central bank credibility. The results for an optimistically inclined public are illustrated in the Figures 6 and 7.

\section{Conclusion}

This paper shows that ambiguity may affect monetary policy in a number of ways. When considering the strategic ambiguity faced by a pessimistically inclined public (the most plausible case), we find that a lack of confidence in the central bank is harmful. It leads to a level of output that is less than its natural rate. This effect is larger when central banks can choose their monetary policy by discretion compared to the case of a rule based monetary policy.

Regarding the state ambiguity faced by central banks, we similarly find that for conservative central banks the impact of state ambiguity is less if monetary policy is guided by credible announcements, rather than being left to the central banks' discretion. 
Finally, we find that ambiguity has no impact on the expected rate of inflation of an optimistic public.

\section{REFERENCES}

[1] Barro, R. And D. Gordon (1983), 'A Positive Theory of Monetary Policy in a Natural Rate Model', Journal of Political Economy, Vol. 91, pp. 589 - 610.

[2] Chprits, E. And B. Schipper (2003), 'Knightian Intransparency of Central Bank and Labor Union', Mimeo, Department of Economics, University of Bonn, Bonn, Germany.

[3] Dow, J. And S. Werlang (1994), 'Nash Equilibrium under Knightian Uncertainty: Breaking Down Backward Induction', Journal of Economic Theory, Vol. 64, pp. 305 324.

[4] Ellsberg, D. (1961), 'Risk, Ambiguity and the Savage Axioms', Quarterly Journal of Economics, Vol. 75, pp. 643 - 669.

[5] Fischer, S. (1977), 'Long-Term Contracts, Rational Expectations and the Optimal Money Supply Rule', Journal of Political Economy, Vol. 85, pp. 191 - 205.

[6] Friedman, M. (1968), 'The Role of Monetary Policy', American Economic Review, Vol. 58 , pp. $1-17$.

[7] Kelsey, D. and W. Spanjers (1997/2004), 'Ambiguity in Partnerships', forthcomming in The Economic Journal.

[8] Knight, F. (1921), Risk, Uncertainty and Profit, Houghton Mifflin, Boston.

[9] Kreps, D. And R. Wilson (1982), 'Reputation and Imperfect Information', Journal of Economic Theory, Vol. 27, pp. 253 - 279.

[10] LuCAs, R. (1976), 'Some International Evidence on Output-Inflation Tradeoffs', American Economic Review, Vol. 63, pp. 326 - 334. 
[11] Marinacci, M. (2000), 'Ambiguous Games', Games and Economic Behaviour, Vol. 31, pp. $191-219$.

[12] Milgrom, P. And J. Roberts (1982), 'Predation, Reputation, and Entry Deterrence', Journal of Economic Theory, Vol. 27, pp. 280 - 312.

[13] Rotemberg, J. (1982), 'Monopolistic Price Adjustment and Aggregate Output', Review of Economic Studies, Vol. 40, pp. 517 - 531.

[14] SchmeIdler, D. (1982/89), 'Subjective Probability and Expected Utility without Additivity', Econometrica, Vol. 5\%, pp. $571-587$.

[15] Spanjers, W. (1999a), Liquiditätsversorgung unter Ungewißheit: Banken oder Märkte?, Habilitation Thesis (in German), University of Saarland, Saarbrücken, Germany.

[16] Spanjers, W . (1999b), 'Loss of Confidence and Currency Crises', Mimeo, The University of Birmingham, Birmingham, United Kingdom.

\section{FiguRES}

Please note that for expositional purposes the location of the line $\pi^{e}=\frac{1}{2}(\underline{\pi}+\bar{\pi})$ is disproportionately to the right in all figures. 


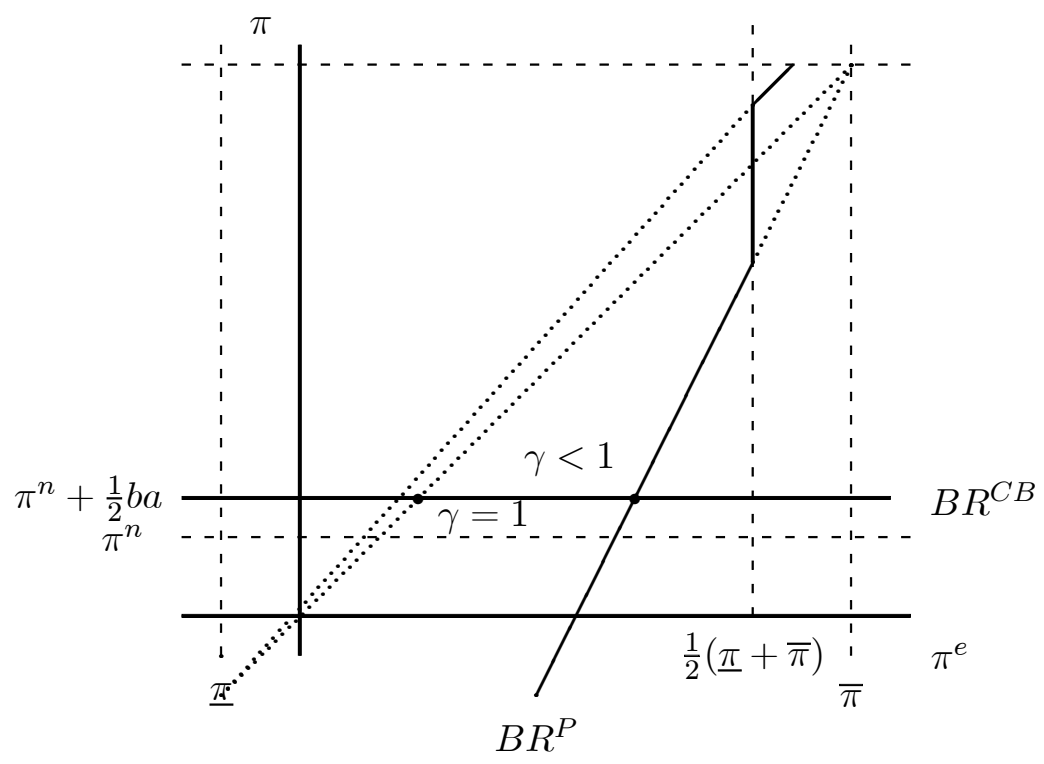

Figure 1: Pessimism: Discretion, $\gamma<1$

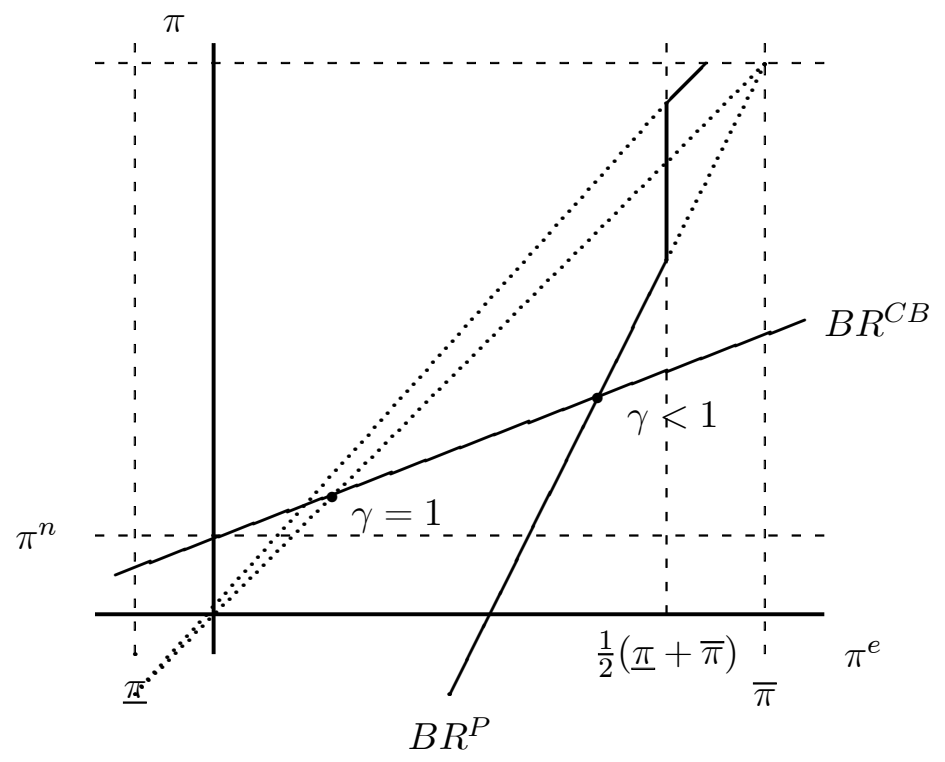

Figure 2: Pessimism: Discretion, Quadratic term 


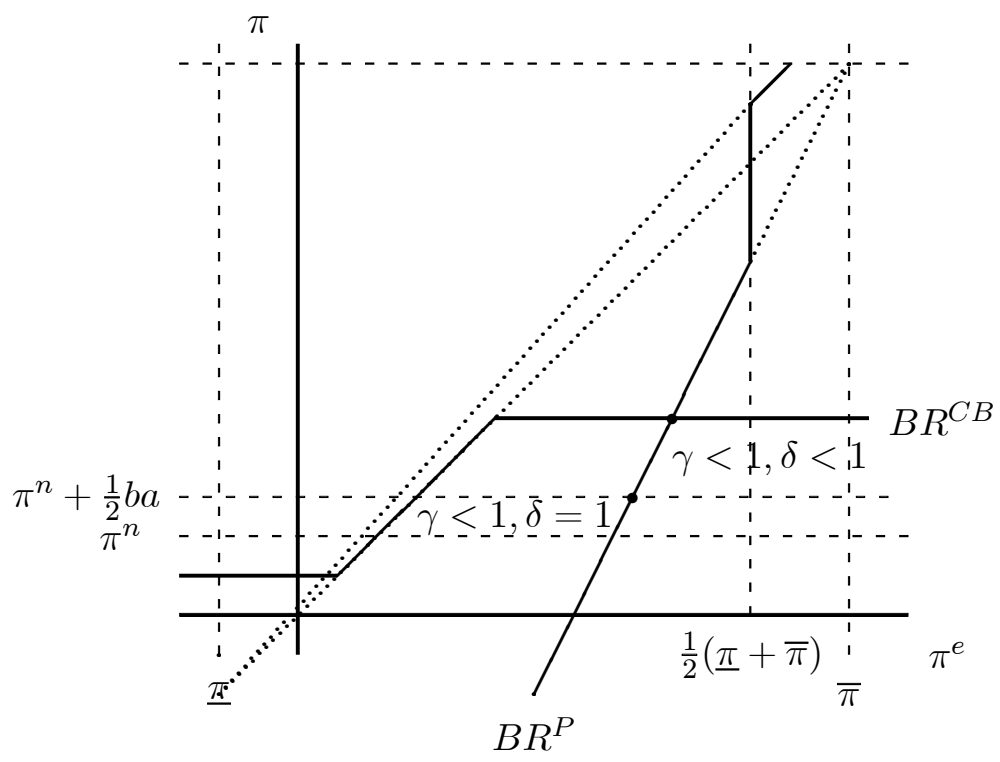

Figure 3: Pessimism: Discretion, $\gamma<1, \delta<1$

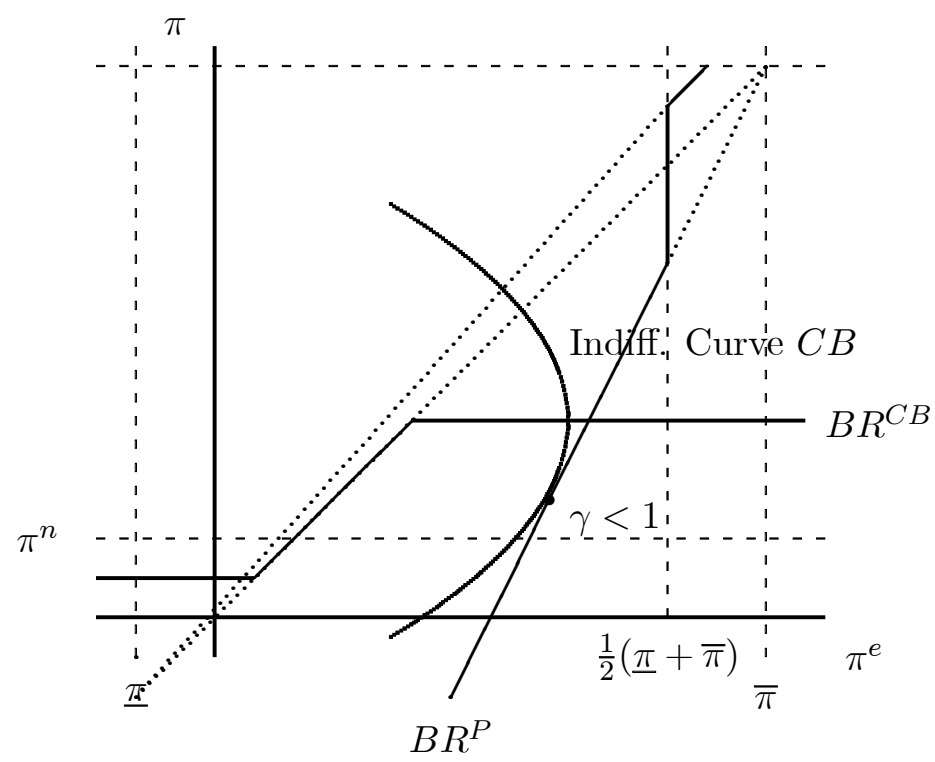

Figure 4: Pessimism: Rules, $\gamma<1$ 


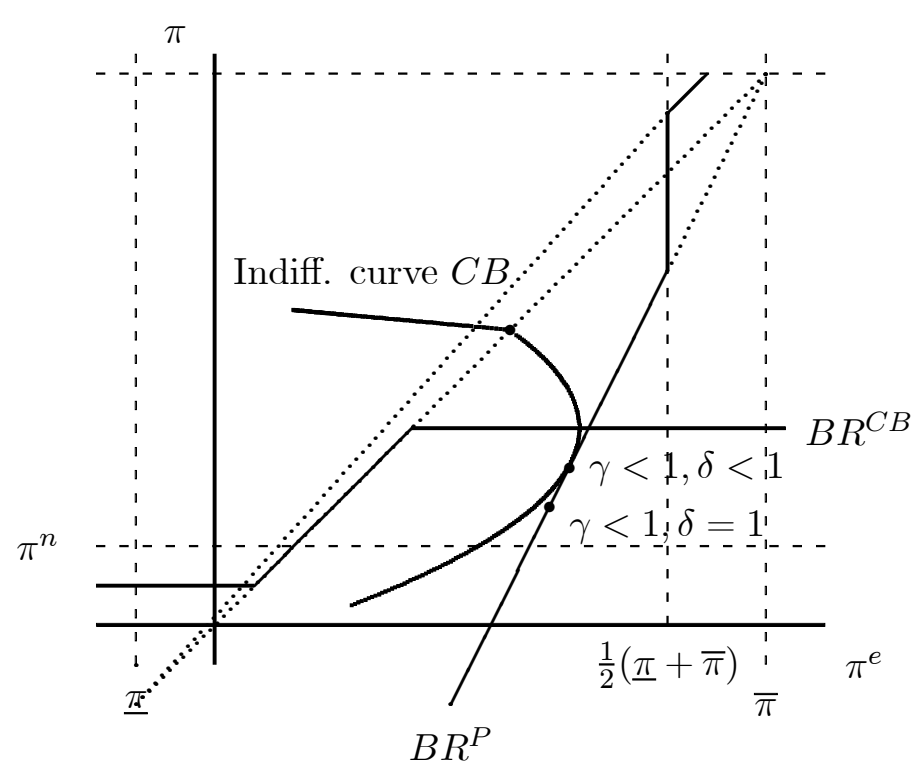

Figure 5: Pessimism: Rules, $\gamma<1, \delta<1$

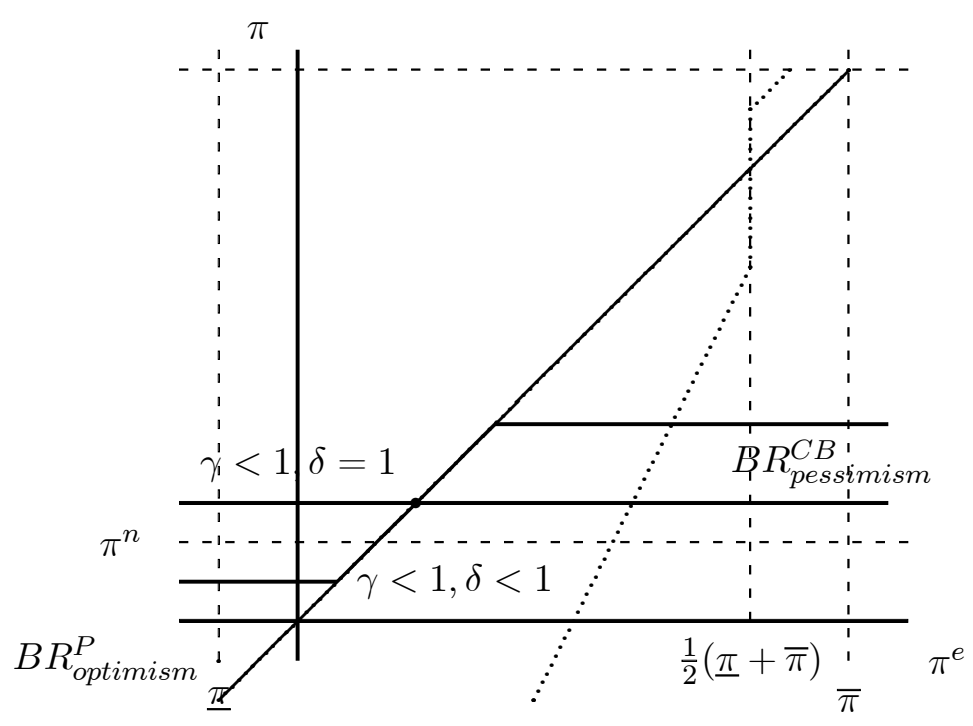

Figure 6: Optimism: Discretion, $\gamma<1, \delta<1$ 


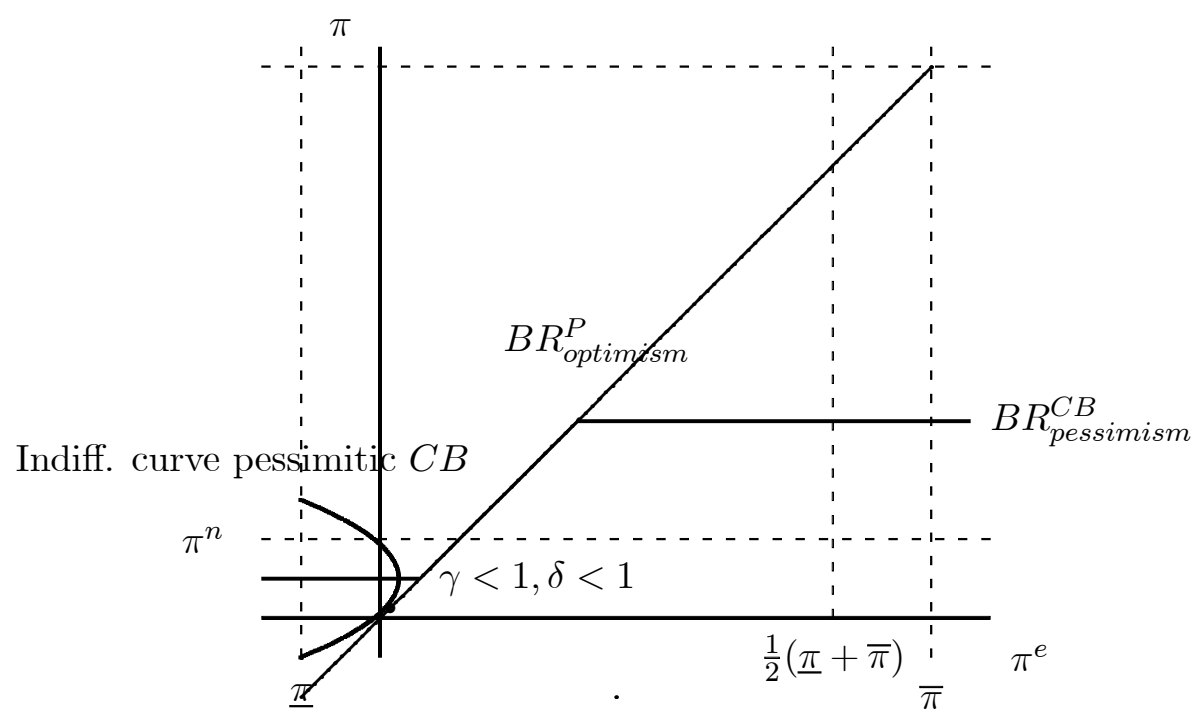

Figure 7: Optimism: Rules, $\gamma<1, \delta<1$ 